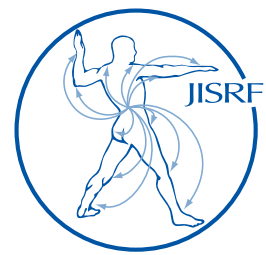

Check for updates

\title{
Robot-assisted Total Hip Arthroplasty After Chiari Pelvic Osteotomy: A Case Report
}

\author{
Dettmer $M^{1}$, Pourmoghaddam $A^{1}$, Kreuzer $S^{1}$
}

\begin{abstract}
Congenital hip dysplasia in younger adults may require surgical treatment. It is believed that 3D-navigated, robotic-assisted surgery for Total Hip Arthroplasty could be useful in cases of pseudo-acetabulum and preceding treatments (Chiari Pelvic osteotomy) due to accurate pre-surgical planning and higher levels of precision associated with the utilized technology, which may improve the odds of positive shortterm and long-term clinical outcomes.

We report the case of a 26-year-old Caucasian woman with a Crowe-IV dysplastic hip and pseudo-acetabulum. Earlier interventions included Chiari pelvic osteotomy, femoral osteotomy and femoral lengthening, which were unsuccessful to improve pain and function in the longer term. The surgical approach via robotic-assisted surgery lead to promising results concerning outcomes in the short-term (four months post-surgery) and medium-term (17 months post-surgery) in this specific case.
\end{abstract}

Keywords: Chiari pelvic osteotomy, robot-assisted surgery, total hip arthroplasty, $3 D$ navigation, hip dysplasia Level of Evidence: AAOS Therapeutic Level IV

\section{Introduction}

Congenital hip dysplasia at different grades (high or low) often requires surgical interventions of Chiari pelvic osteotomy and/or Total Hip Arthroplasty (THA) early in life [1]. Since bone-preservation at early stages of life decreases the odds of potential revision surgeries, it has been suggested that Chiari pelvic osteotomy may be a more appropriate treatment approach in younger patients to conserve the hip joint compared to a THA [2].

However, when THA becomes necessary in patients having previously undergone Chiari pelvic osteotomy or other interventions, pre-operative planning may become difficult because the pseudo-acetabulum is significantly different from the true acetabulum and deformities related to congenital hip dysplasia may result in additional intra- operation complexities. Additionally, determination of the true leg length can be challenging in such cases.

Here, we report the case of a 3-dimensional (3-D) robotic-assisted THA for a Crowe-IV dysplastic hip with pseudo-acetabulum that had previous surgical interventions of Chiari pelvic osteotomy, femoral osteoplasty, and femoral lengthening. We decided to use the MAKOplas-

\footnotetext{
1 Marius Dettmer, PhD; Amir Pourmoghaddam, PhD; Stefan W Kreuzer, PhD Memorial Bone \& Joint Research Foundation, 1140 Business Center Drive Suite 101, 77043 Houston, TX (Direct reprint requests to Marius Dettmer)
}

(C) 2016 Dettmer, Pourmoghaddam, Kreuzer. All rights reserved.

Reconstructive Review is a peer-reviewed, open-access orthopaedic open 6 Access journal devoted to publishing papers in the area of reconstructive arthroplasty. Authors retain copyright and grant the journal right of first publication with the work. Reconstructive Review follows the Creative Commons AttributionNonCommercial CC BY-NC. This license allows anyone to download works, build upon the material, and share them with others for non-commercial purposes as long as they credit the senior author, Reconstructive Review, and the Joint Implant Surgery \& Research Foundation (JISRF). 
ty $\mathrm{RIO}^{\circledR}$ robotic arm (MAKO Surgical Corp., Ft. Lauderdale, FL) since we expected that utilizing this technology would enable us to pre-operatively plan the placement of the acetabular shell into the severely dysplastic true acetabulum by creating a patient-specific surgical plan (based on 3D reconstructions of the patient's CT scans). Additionally, we expected to be able to execute this plan with high levels of positional precision and orientation accuracy. We hypothesized that this treatment would lead to good implant alignment, which would result in a reduction of the risk for complications and an improvement of the patient's Hip disability and Osteoarthritis Outcome Score (HOOS) and UCLA activity scores post-operatively in the short and medium term.

\section{Case Presentation}

The patient was a 26-year-old Caucasian female who weighed 130 pounds $(59 \mathrm{Kg})$ and stood 67 inches tall $(B M I=20)$. She did not report any significant past medical issues besides congenital hip dysplasia with symptoms initially appearing at age 12 , requiring multiple procedures. In 2001, she had undergone a Chiari pelvic osteotomy to help relieve her pain from Crowe-IV dysplasia. Subsequently, the patient had femoral osteotomy and femoral lengthening in 2005. These interventions only partially corrected her $60 \mathrm{~mm}$ leg length discrepancy and $35 \mathrm{~mm}$ radiologic alignment discrepancy. She reported that her pain improved for several years, but the treatments caused formation of pseudo-acetabulum of the right hip and reestablished a leg length discrepancy (Figure 1). Over time, the pain returned with no significant relief from cortisone injections or oral analgesics.

Radiographic images of the right hip showed end-stage osteoarthritis and global joint space narrowing. The im-

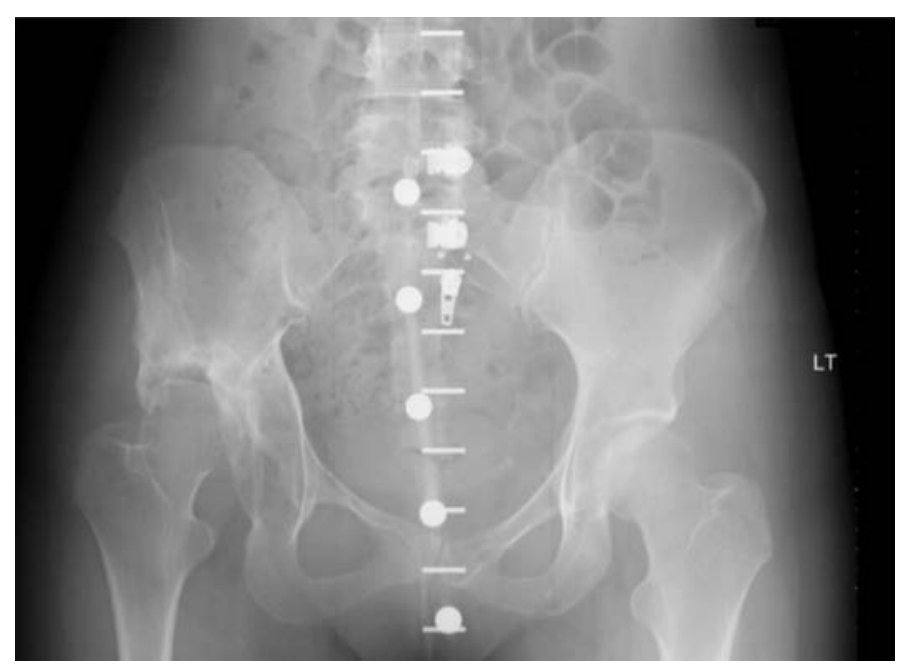

Figure 1.Pre-operative X-ray of 26-year old patient with severe hip dysplasia and pseudo-acetabulum ages also indicated formation of osteophytes on the lateral margin and medial wall of the pseudo-acetabulum, as well as on the femoral head as shown in Figure 1. At this point, we decided to plan and perform a THA using robotic-assisted surgery to reconstruct the normal (true) acetabulum (Figure 2) and to implant a Corin ${ }^{\circledR}$ MiniHip (Corin Group, Circester, United Kingdom) neck preserving hip stem.

Pre-operatively, the patient completed a survey that included HOOS and UCLA- activity questionnaires. These surveys are designed to evaluate patients' subjective opinion concerning their hip, associated issues, symptoms and functional impairments. The HOOS survey consists of several sub- sections designed to assess severity of osteoarthritis. These sections are: 1) symptoms, 2) pain, 3) activity of daily living (ADLs), 4) sports and recreation, and 5) quality of life (QOL) [3]. Additionally, the patient indicated that her pain represented a serious limitation and she was unable to perform ordinary functions. She also stated that her limp was moderate and she was only able to walk indoors. The patient's UCLA activity score indicated that she was mostly physically inactive and severely limited regarding activities of daily living.

\section{Surgical Treatment}

In the initial phase of the surgical planning, typically CT-scans and X-rays are used to plan alignment of the implants, associated anteversion (Figure 2a), inclination (Figure $2 \mathrm{~b}$ ), and positioning (figure $2 \mathrm{c}$ ).

We supplemented this planning stage with a 3-D reconstructed model based on CT-scans. This model could be freely rotated in all directions and was used for inspection of the involved structures and planning of positioning, anteversion, inclination, and optimal bone coverage (see Figure 3).

The navigation system preparation and the surgical technique via a direct anterior approach have been described in detail before [4]. The anterior approach was used because it facilitates computer navigation/robotics in the supine position. Furthermore, this patient had undergone a SmithPetersen (anterior approach) procedure performed during previous surgery, and the table extension used in this approach assisted in reducing the hip due to the required significant leg lengthening.

The patient was placed in supine position on the operative table with the operative leg attached to the arch table extension (Innovative Orthopedic Technologies LLC, Houston, TX). The procedure was performed using MAKOplasty and a RIO Robotic Arm Interactive Orthopedic System (MAKO Surgical Corp, Ft. Lauderdale, FL 


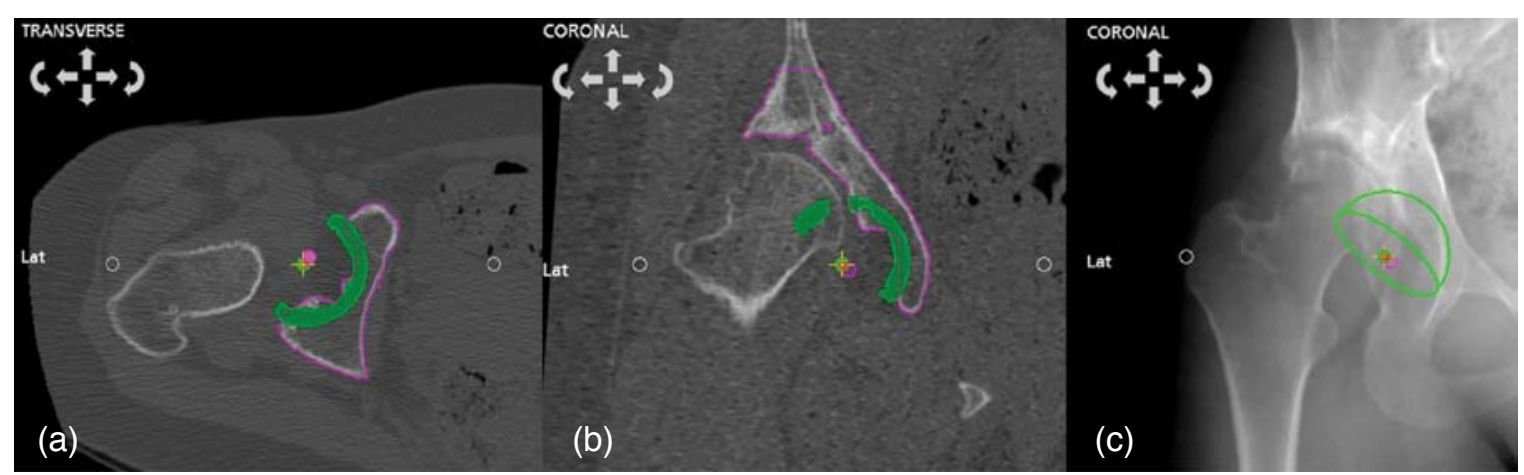

Figure 2. (a) CT-scan, transverse view of the hip including planned acetabulum shell, $(b)$ CT-scan, coronal view of planned acetabulum shell (c) $x$-ray coronal view of planned acetabulum position.
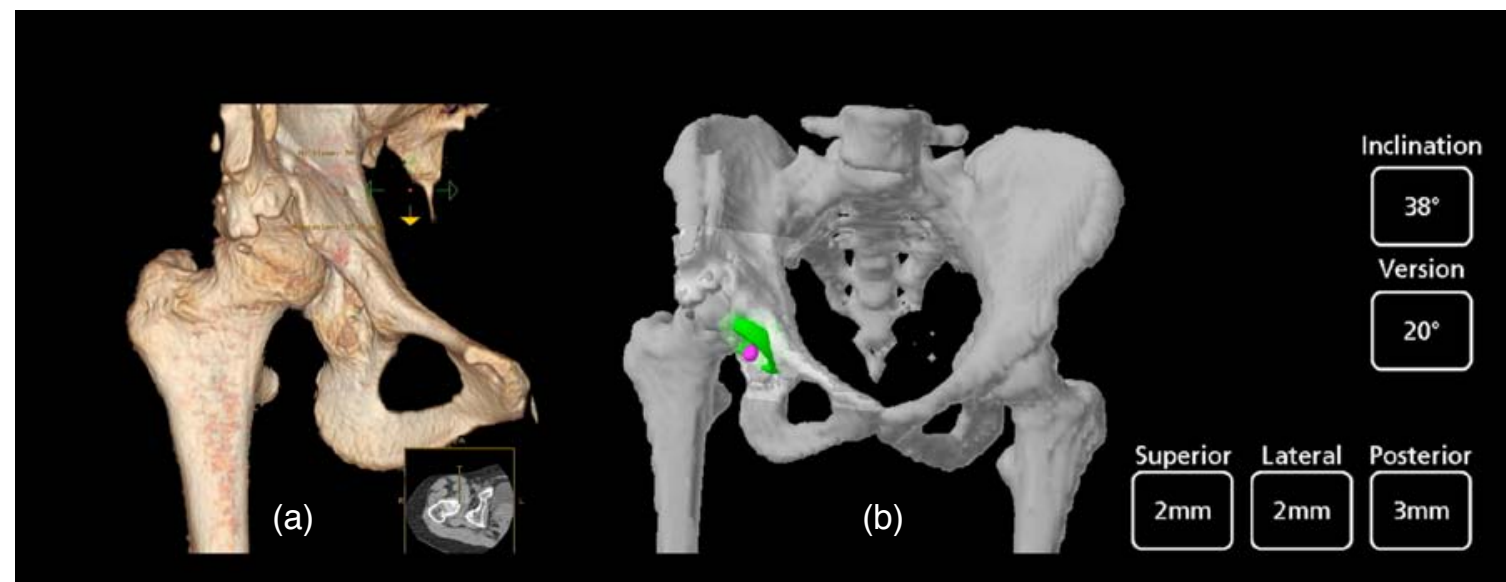

Figure 3. (a) 3-D reconstruction of the hip including pseudoacetabulum, (b) 3-D reconstruction of the hip including planned cup position

USA). Initially, pelvic trackers were placed into the pelvic bone on the left iliac crest (contralateral hip) utilizing two pins and the registration was performed after surgical exposure of the hip joined was completed. A small reference check point was placed into the proximal femur as well as an ECG lead on the knee cap of the operative leg as a reference points for measuring leg length and offset. Once the femoral neck osteotomy was complete according to the pre-operative plan, the true acetabulum was registered. Visual inspection of the joint during the surgery confirmed pre-operative assessments; the operative findings were consistent with end-stage osteoarthritis disease and significant synovitis. The femoral head and acetabular surface were noted to be deformed with bone on bone articulation. Additionally, there was a significant amount of osteophytes on the femoral neck and acetabular rim of the pseudo-acetabulum.

An initial reaming one size smaller of the final cup size (42mm) was completed. Visual inspection and finger palpation was utilized to assure maintenance of the anterior and posterior wall. At this time the final reamer $(44 \mathrm{~mm})$ was placed again utilizing the robotic arm to complete the reaming. A size $44 \mathrm{~mm}$ Corin ${ }^{\circledR}$ Trinity ${ }^{\mathrm{TM}}$ cup (Corin Group, Circester, United Kingdom) was implanted using the robotic arm. Two additional screws were utilized for additional fixation due to the dysplastic nature of the acetabulum. A 10 degree lipped liner was then impacted into the acetabulum shell for additional posterior stability. Af- ter preparation of the femoral side, a size 3 standard hydroxyapatite coated MiniHip ${ }^{\mathrm{TM}}$ stem, with a $28 \mathrm{~mm}$ diameter femoral head was implanted. After this trial reduction, the hip was found to be stable in flexion, extension, internal and external rotation, with satisfactory restoration of the leg length (Figure 4) according the the preoperative leg length inequality $(13 \mathrm{~mm})$.

The surgery led to the reconstruction of a normal acetabulum (true acetabulum), which lead to a decrease in clinical leg length discrepancy, as determined via measurement during the surgery. The patient was partially weightbearing for the first 4 weeks after surgery with no dislocation precautions in place. The patient was followed up at 4 months and 17 months postoperatively. She demonstrated significant improvement in all clinical scores after 4 months and even further improvements after 17 months, as illustrated in Table 1. She indicated that she resumed all her daily activities and has returned to sports.

\section{Discussion}

Robot-assisted surgery has become a commonly used tool in hip and knee Arthroplasty over the last two decades [5-7] due to the associated advantages of precision of bone preparation, high accuracy of pre-surgery planning and improved navigation in hip Arthroplasty [8,9]. While these advantages have been described in general population set- 


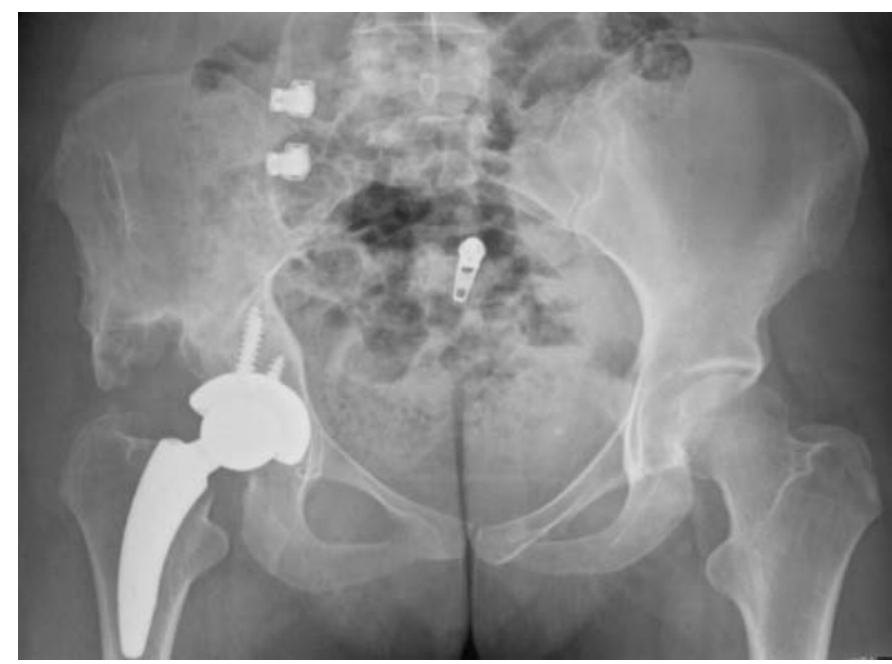

Figure 4. Post-surgery $x$-ray of 26-year old patient with implanted acetabulum shell and Corinß MiniHip ${ }^{T M}$.

\begin{tabular}{|l|l|c|c|c|c|}
\hline & & $\begin{array}{c}\text { Pre- } \\
\text { op }\end{array}$ & $\begin{array}{c}\text { Post-op } \\
\text { (4 months) }\end{array}$ & $\begin{array}{c}\text { Post-op } \\
(\mathbf{1 7} \text { months })\end{array}$ & $\begin{array}{c}\text { Change } \\
\text { score }\end{array}$ \\
\hline HOOS & Symptoms & 35 & 65 & 90 & +55 \\
\hline & Pain & 58 & 90 & 100 & +42 \\
\hline & ADL & 61 & 93 & 98 & +37 \\
\hline & Sports & 32 & 63 & 82 & +50 \\
\hline UCLA & QOL & 13 & 75 & 88 & +75 \\
\hline
\end{tabular}

Table 1. Results from subcategories of the HOOS and UCLA Activity Score before, 4 months/17 months after surgery and score change between pre-op and 17 months post-op. Maximum HOOS is 100, Maximum UCLA Activity Score is 10.

tings, we believe it is useful to report the outcome from patients with advanced anatomical deformity and special needs, such as in the current case. This will enable clinicians to determine the technology's capability for improving clinical outcomes in a variety of cases and in the longer-term, and to evaluate longevity of robotic-assisted surgery. Specifically, evidence from either clinical trials or case studies is required to justify the added costs associated with this type of surgery [10].

In the current case, traditional surgery would have been challenging for the team without robotic assistance. The approach presented here was effective due to precise planning of the new acetabulum and placement of the MiniHip. Using this technology, the surgeon was provided with accurate quantitative knowledge about current position of the instrument and alignment of anatomical structures. This allowed for high accuracy in this case of severe dysplasia and deformation, whereas the robotic arm provided a failsafe mechanism against manual errors based on haptic feedback.
The success was reflected in the improvement of the patient's self-reported outcomes, both in the shorter-term (four months) and mid-term (17 months). More specifically, the surgical treatment improved pain measures and functional outcomes considerably and allowed the patient to quickly return to pain-free, moderate activity.

\section{Duration of Surgery}

In a series of robotically assisted total hip replacements, after the learning curve, the average operative time is approximately 15 minutes longer than a navigated total hip replacement. Placement of the trackers is about 3 minutes and the registration process is about 10 minutes. In this particular case, the false acetabulum was marked as the acetabulum and during the case the three orientation points needed to be changed, which added another 15 minutes to the surgery. It is difficult to say if the robotic surgery extended the surgical time, but the acetabular prep time (reaming and placement of the socket) was less than 10 minutes. Overall, in the surgeon's estimate, the surgical time was extended by approximately 30 minutes and the overall surgical time was 120 minutes skin to skin with EBL of $450 \mathrm{cc}$, with $155 \mathrm{cc}$ given back from the cell saver. Part of the increased surgical time was not due to robotic utilization but due to the surgeon not using additional screws for acetabular fixation initially, which caused the socket to move after the initial reduction. This was clearly a misjudgment by the surgeon, as he considered the initial press fit to be excellent. The cup was then repositioned and three screws were placed for additional fixation. This being a very unique and complex case in a very young patient, the surgeon felt obligated to assure optimal implant position to avoid any potential complication in the future. Although the surgeon is considered an expert in hip replacement, he does not have extensive experience regarding displastic hips with previously conducted osteotomies.

\section{Additional cost of Robotic-Assisted Surgery}

At this point, it is very difficult to determine the cost effectiveness of this procedure. Obviously, it is not cost effective if a robot is purchased only for this type of surgeries or even for general hip replacement. In fact, the operating surgeon in this case no longer uses the robot in routine total hip replacements, since outcome results from our clinic did not differ regarding robot-assisted surgery and "regular" navigated total hip replacement surgery. Therefore, robotic assistance was not considered cost effective. 
The main reason to present this case is that this particular technology could be valuable in complex cases such as the presented one if the technology is available in the hospital.

\section{Conclusion}

To our knowledge, this is the first time a case of a patient suffering from Crowe-IV hip dysplasia with pseudoacetabulum and robotic-assisted THA is reported, whereas previous surgical interventions included Chiari pelvic osteotomy, femoral osteotomy and femoral lengthening. Robotic-assisted, 3-D navigated hip Arthroplasty showed promising results in the current patient. A THA operation (in severely deformed hips) can be safely performed with robotic assistance and a good outcome can be achieved. However, it is not yet clear if robotic assistance will lead to consistently similar outcomes compared to traditional techniques in specific cases such as the one presented here. We have since utilized this approach in cases of previous acetabular fracture to predict the potential need for hardware removal. More research is needed to shed light on benefits or potential limitations of the technology in other specific patient cases. Additionally, it is required to investigate the long-term outcomes resulting from this intervention, specifically in younger patients.

\section{Acknowledgements}

The authors would like to thank Mr. Brian Caballero for his assistance in the data collection and Mr. David Balderree for assistance in managing the collected data. Additionally, the authors would like to thank Dr. Ashish Arya for his assistance during the editing phase of this paper and his provision of valuable feedback.

\section{Disclosure}

The authors declare that there is no conflict of interest regarding the publication of this paper. For full disclosures refer to last page of this journal.

\section{References}

1. Troelsen A. Assessment of adult hip dysplasia and the outcome of surgical treatment. Dan Med J 2012;59:B4450.

2. Ito $\mathrm{H}$, Matsuno T, Minami A. Chiari pelvic osteotomy for advanced osteoarthritis in patients with hip dysplasia. J Bone Joint Surg Am 2004;86-A:1439-45.

3. Nilsdotter AK, Lohmander LS, Klässbo M, Roos EM. Hip disability and osteoarthritis outcome score (HOOS)--validity and responsiveness in total hip replacement. BMC Musculoskelet Disord 2003;4:10. doi:10.1186/1471-2474-4-10.

4. Kreuzer S, Leffers K. Direct anterior approach to total hip Arthroplasty using computer navigation. Bull NYU Hosp Jt Dis 2011;69:S52-5.

5. Conditt MA, Bargar WL, Cobb JP, Dorr LD, Lonner JH. Current concepts in robotics for the treatment of joint disease. Adv Orthop 2013;2013:948360. doi:10.1155/2013/948360.

6. Lang JE, Mannava S, Floyd a J, Goddard MS, Smith BP, Mofidi a, et al. Robotic systems in orthopaedic surgery. J Bone Joint Surg Br 2011;93:1296-9. doi:10.1302/0301-620X.93B10.27418.

7. Ponnusamy K, Golish S. Robotic Surgery in Arthroplasty. AAOS Now 2013;7:36.

8. Tarwala R, Dorr LD. Robotic assisted total hip Arthroplasty using the MAKO platform. Curr Rev Musculoskelet Med 2011;4:151-6. doi:10.1007/s12178-0119086-7.

9. Dorr LD, Jones RE, Padgett DE, Pagnano M, Ranawat AS, Trousdale RT. Robotic guidance in total hip Arthroplasty: the shape of things to come. Orthopedics 2011;34:e652-5. doi:10.3928/01477447-20110714-30.

10. Werner SD, Stonestreet M, Jacofsky DJ. Makoplasty and the Accuracy and Efficacy of Robotic-assisted Arthroplasty. Surg Technol Int 2014;24:302-6. 